\title{
PAPER
}

\section{Implementation of initial and boundary conditions in the finite-difference time-domain numerical solution of elastic wave fields in anisotropic solids}

\author{
Masahiro Sato* \\ Faculty of Education and Human Studies, Akita University, \\ 1-1, Gakuencyo, Tegata, Akita, 010-8502 Japan \\ (Received 27 March 2005, Accepted for publication 1 August 2005)
}

\begin{abstract}
Methods of treating initial and boundary conditions when applying the finite difference time domain (FDTD) numerical method to the analysis of elastic wave fields in anisotropic solids have been considered with reference to an electrical circuit analogy. It was found that the staggered lattice of the FDTD analysis of anisotropic solids is expressed by the superposition of two electric circuit networks of identical configurations. One of these circuits is translated relative to the other by one grid length in both the $y$ and $z$ directions, and is then superposed onto the other circuit to yield the final overall circuit. The two circuits are connected by the mutual capacitance between the capacitances loaded at the normal stress nodes of one of the circuits, and the shear stress nodes of the other circuit, which are positioned on the same stress nodes. From the configurations of the circuits, it can be concluded that the initial and boundary conditions should be applied to both the circuit networks. The theory was confirmed by considering two model problems.
\end{abstract}

Keywords: FDTD method, Anisotropic solids, Elastic waves, Numerical analysis, Time domain

PACS number: 43.20.Px, 43.35.Cg, 43.35.Pt, 43.35.Zc [DOI: 10.1250/ast.27.36]

\section{INTRODUCTION}

Sato and coworkers have previously investigated the application of the FDTD numerical method to the analysis of time domain problems of elastic wave fields [1-6]. However, only isotropic solids are considered in their studies. For this reason, in a recent study, Sato attempted to obtain a formulation of the FDTD method appropriate for the analysis of two-dimensional elastic wave fields in anisotropic solids [7]. The staggered lattice network used in this study differs from that for an isotropic solid only in the requirement of two variables on a particle velocity node and three variables on a stress node, in contrast to the isotropic case of one variable on a particle velocity node and one or two variables on a stress node. Moreover, in the case of anisotropic solids, all discretized stress components appear on each stress node, and also all elements of the discretized particle velocity vector appear on each particle velocity node. This situation has not previously been encountered in the implementation of staggered lattice networks, and thus, methods of implementing initial and boundary conditions are unclear.

In this study, we employ an electrical circuit analogy of

*e-mail: ma-sato@ed.akita-u.ac.jp the elastic wave fields in order to clarify the methods that may be used to apply the boundary conditions. It is shown that the staggered lattice for an anisotropic solid is expressed by the superposition of two electrical circuit networks, similar to that in the case of an isotropic solid. These two circuits are connected by the mutual capacitance between the normal stress $T_{2}$ nodes of one circuit and the shear stress $T_{4}$ nodes of the other circuit, which are located at the same lattice positions.

Using the analogy, we conclude that we must set the initial and boundary conditions on both the two circuits. In this report, the author introduces methods of implementing input signals and boundary conditions, particulaly for the free-boundary conditions that frequently occur in elastic wave field problems.

\section{FUNDAMENTAL ELASTIC EQUATIONS OF Y-Z PLANE OF QUARTZ}

We consider the two-dimensional surface strain problem on the $Y-Z$ crystallographic plane of quartz that has trigonal crystal classes with $32,3 m$, and $3 \bar{m}$. Although Yamada and Sato have previously established the FDTD formulation for piezoelectric materials [2], in the present study, the piezoelectric effect of quartz is omitted for a clear and simple discussion. 
The fundamental equations are expressed as

$\frac{\partial}{\partial t}\left[\begin{array}{c}T_{2} \\ T_{3} \\ T_{4}\end{array}\right]=-\left[\begin{array}{ccc}c_{22} & c_{23} & -c_{24} \\ c_{23} & c_{33} & 0 \\ -c_{24} & 0 & c_{44}\end{array}\right] \cdot\left[\begin{array}{c}\frac{\partial \dot{v}}{\partial y} \\ \frac{\partial \dot{w}}{\partial z} \\ \frac{\partial \dot{v}}{\partial z}+\frac{\partial \dot{w}}{\partial y}\end{array}\right]$,

$\rho \frac{\partial}{\partial t}\left[\begin{array}{c}\dot{v} \\ \dot{w}\end{array}\right]=\left[\begin{array}{l}\frac{\partial T_{2}}{\partial y}+\frac{\partial T_{4}}{\partial z} \\ \frac{\partial T_{3}}{\partial z}+\frac{\partial T_{4}}{\partial y}\end{array}\right]$,

in which the coordinate axes $y$ and $z$ are coincident with the crystallographic axes $Y$ and $Z$, respectively. Equation (1) describes Hooke's law, while Eq. (2) expresses Newton's second law of motion [8]. Here, $\dot{v}$ and $\dot{w}$ are particle velocities in the $y$ and $z$ directions, respectively. The variables $T_{2}$ and $T_{3}$ are normal stresses, and $T_{4}$ is a shear stress. The stress vector and stress tensor are related by $T_{2}=T_{y y}$, $T_{3}=T_{z z}$, and $T_{4}=T_{y z},[c]$ is a stiffness matrix, and $\rho$ denotes mass density.

\section{EQUIVALENT ELECTRICAL CIRCUIT OF ELASTIC WAVE FIELD ON $Y-Z$ PLANE OF QUARTZ}

In this section, we consider the physical meaning of the staggered lattice network, derived in [7], using an electrical analogy.

Figure 1(a) shows the distribution of the discrete variables of elastic waves for an anisotropic solid. This staggered lattice is fundamental to the numerical formulation of the problem by leap-frog finite-difference approximation. Note that the lattice shown in Fig. 1(a) differs from the conventional discretization for isotropic solids shown in Fig. 1(b) [1].

Using the compliance matrix [s], Eq. (1) is written as

$$
\left[\begin{array}{ccc}
s_{22} & s_{23} & -s_{24} \\
s_{23} & s_{33} & 0 \\
-s_{24} & 0 & s_{44}
\end{array}\right] \frac{\partial}{\partial t}\left[\begin{array}{c}
T_{2} \\
T_{3} \\
T_{4}
\end{array}\right]=\left[\begin{array}{c}
\frac{\partial \dot{v}}{\partial y} \\
\frac{\partial \dot{w}}{\partial z} \\
\frac{\partial \dot{v}}{\partial z}+\frac{\partial \dot{w}}{\partial y}
\end{array}\right],
$$

where $s_{i j}(i, j: 2,3,4)$ is the $i, j t h$ element of the compliance matrix $[s]\left(=[c]^{-1}\right)$. Noted that Eqs. (2) and (3) closely resemble the no-loss telegraphic equation shown in Eq. (4), where $V$ and $I$ denote the electrical voltage and current, respectively, and the circuit is spatially one-dimensional,

$$
\left.\begin{array}{c}
L \frac{\partial I}{\partial t}=-\frac{\partial V}{\partial x} \\
C \frac{\partial V}{\partial t}=-\frac{\partial I}{\partial x}
\end{array}\right\} .
$$

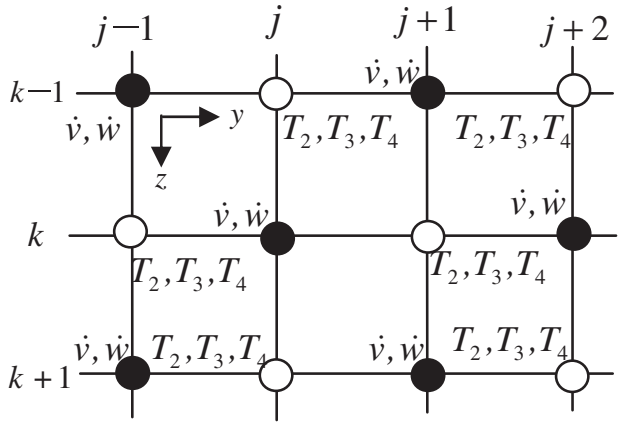

(a)

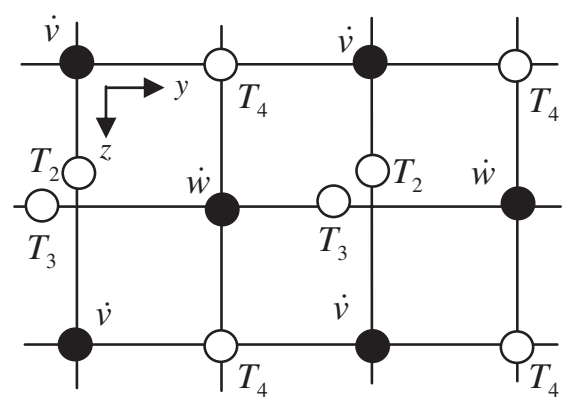

(b)

Fig. 1 Staggered lattice network for the numerical solution of the elastic wave field of an anisotropic solid. (a) The distribution of the discrete variables of elastic waves for an anisotropic solid. (b) The conventional discretization for isotropic solids.

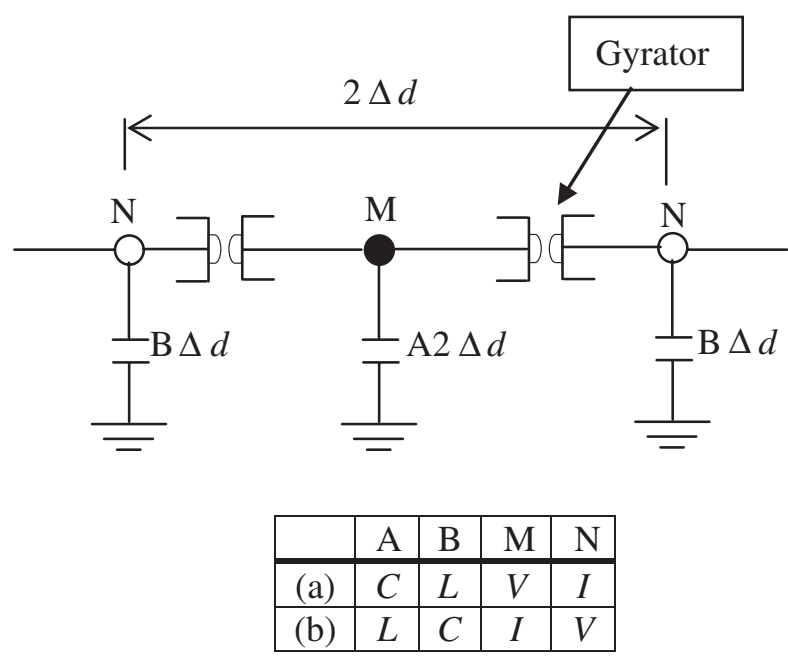

Fig. 2 Fundamental part of a distributed electric circuit. (a) $\mathrm{T}$ type of the distributed circuit, (b) $\pi$ type of the distributed circuit.

The centered finite-difference approximation of the spatial derivatives in Eq. (4) yields the fundamental section of the circuit network shown in Fig. 2. In the same manner, we obtain the fundamental section of the equivalent circuit network, shown in Fig. 3, for the elastic wave field derived using Eqs. (2) and (3).

By observing the circuit, it is clear that the staggered 


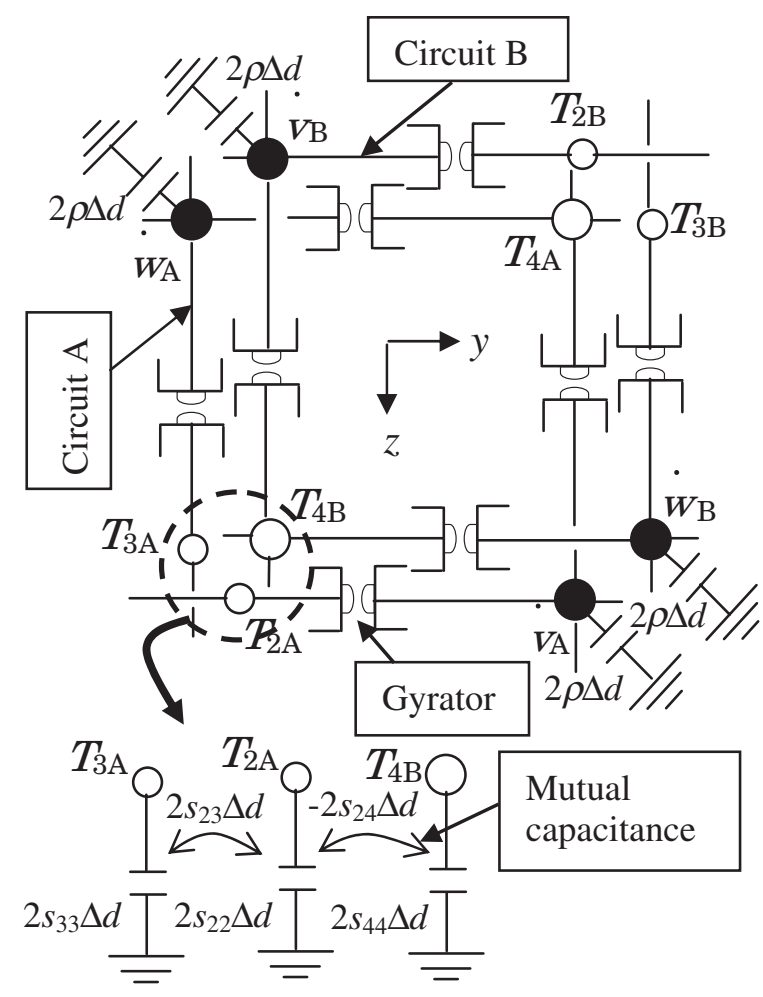

Fig. 3 Fundamental part of the equivalent circuit network of the elastic wave fields on the $Y-Z$ plane of quartz.

lattice for the elastic waves of an anisotropic solid (quartz) is expressed by the superposition of two electrical circuit networks (named A and B in Fig. 3), and the capacitance of the $T_{2}$ node of one circuit and that of the $T_{4}$ node of the other circuit are connected by a mutual capacitance on each stress node. On the $T_{2}$ and $T_{3}$ stress nodes $(j+1, k)$ of circuit A, and the $T_{4}$ node of circuit B shown in Fig. 4 , the following equations are derived using Kirchhoff's law,

$\dot{v}_{A}(j+2, k)-\dot{v}_{A}(j, k)=s_{11} 2 \Delta d \frac{\partial T_{2 \mathrm{~A}}(j+1, k)}{\partial t}$

$$
\begin{aligned}
& \quad+s_{33} 2 \Delta d \frac{\partial T_{3 \mathrm{~A}}(j+1, k)}{\partial t}-s_{24} 2 \Delta d \frac{\partial T_{4 \mathrm{~B}}(j+1, k)}{\partial t}, \\
& \dot{w}_{A}(j+1, k+1)-\dot{w}_{A}(j+1, k-1) \\
& \quad=s_{11} 2 \Delta d \frac{\partial T_{2 \mathrm{~A}}(j+1, k)}{\partial t}+s_{33} 2 \Delta d \frac{\partial T_{3 \mathrm{~A}}(j+1, k)}{\partial t}, \\
& \dot{v}_{B}(j+1, k+1)-\dot{v}_{B}(j+1, k-1) \\
& \quad+\dot{w}_{B}(j+2, k)-\dot{w}_{B}(j, k)=s_{11} 2 \Delta d \frac{\partial T_{2 \mathrm{~A}}(j+1, k)}{\partial t} \\
& \quad-s_{24} 2 \Delta d \frac{\partial T_{4 \mathrm{~B}}(j+1, k)}{\partial t},
\end{aligned}
$$

where $\Delta y=\Delta z=\Delta d$ is assumed. In a similar manner, sets of equations at the nodes $(j+2, k+1)$ are obtained, with the average of two sets of equations converging to Eq. (3) in the limit as $\Delta d \rightarrow 0$.

Thus, it is clear that we should treat boundary conditions by applying them to both the circuits. With regard to the method of treating initial conditions, however, we can select from the two methods. The first method is formulated by applying the same conditions to both the circuits. For this case, it would be unnecessary to average the values of the two circuits. The second method is formulated by applying an initial condition to only one of the circuits; thus, no initial condition is loaded on the other circuit. This method would require the numerical results of the two circuits to be averaged in order to obtain a valid numerical approximation. Furthermore, the amplitude of the initial values should be twice those used in the first method.

\section{RELATIONSHIP BETWEEN FORMULATION BY FDTD METHOD AND EQUIVALENT CIRCUIT}

With reference to the staggered lattice of circuit A shown in Fig. 4, the first equation of Eq. (1) is formulated by the FDTD method as

\section{Circuit A}

\section{Circuit B}

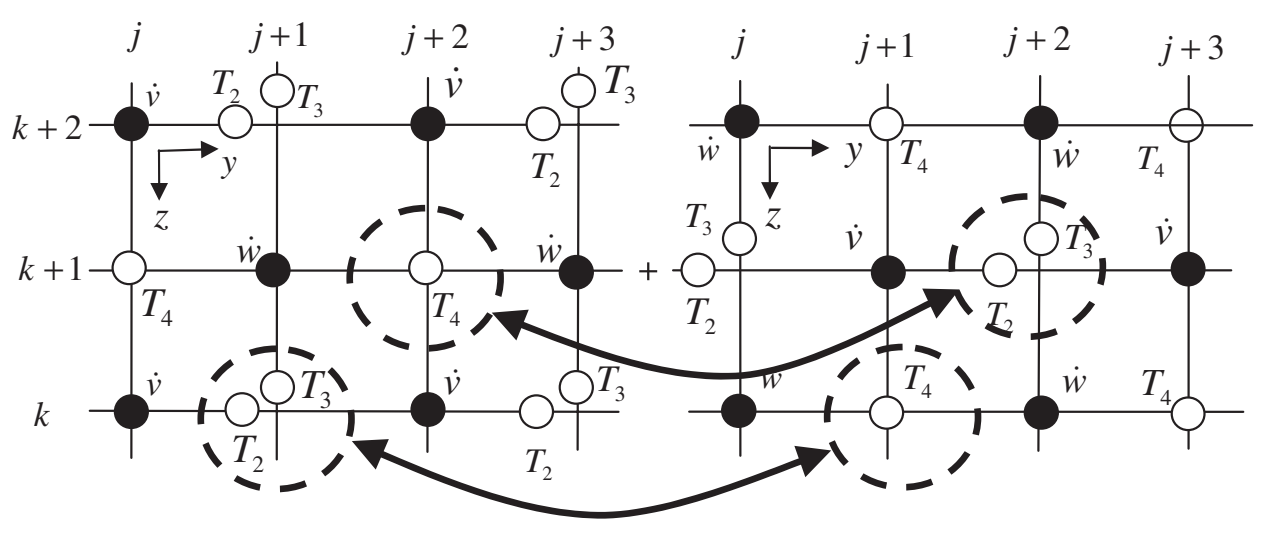

Fig. 4 Superposition of circuits A and B. 


$$
\begin{aligned}
\frac{2 \Delta d}{2 \Delta t} & \left\{T_{2 \mathrm{~A}}^{n+1}(j+1, k)-T_{2 \mathrm{~A}}^{n-1}(j+1, k)\right\} \\
= & c_{22}\left\{\dot{v}_{\mathrm{A}}^{n}(j+2, k)-\dot{v}_{\mathrm{A}}^{n}(j, k)\right\} \\
& +c_{23}\left\{\dot{w}_{\mathrm{A}}^{n}(j+1, k+1)-\dot{w}_{\mathrm{A}}^{n}(j+1, k-1)\right\} \\
& -c_{24}\left\{\dot{v}_{\mathrm{B}}^{n}(j+1, k+1)-\dot{v}_{\mathrm{B}}^{n}(j+1, k-1)\right\} \\
& -c_{24}\left\{\dot{w}_{\mathrm{B}}^{n}(j+2, k)-\dot{w}_{\mathrm{B}}{ }^{n}(j, k)\right\},
\end{aligned}
$$

while with reference to that of circuit B in Fig. 4, we derive

$$
\begin{aligned}
\frac{2 \Delta d}{2 \Delta t} & \left\{T_{2 \mathrm{~B}}^{n+1}(j+2, k+1)-T_{2 \mathrm{~B}}^{n-1}(j+2, k+1)\right\} \\
= & c_{22}\left\{\dot{v}_{\mathrm{B}}{ }^{n}(j+3, k+1)-\dot{v}_{\mathrm{B}}{ }^{n}(j+1, k+1)\right\} \\
& +c_{23}\left\{\dot{w}_{\mathrm{B}}{ }^{n}(j+2, k+2)-\dot{w}_{\mathrm{B}}{ }^{n}(j+2, k)\right\} \\
& -c_{24}\left\{\dot{v}_{\mathrm{A}}^{n}(j+2, k+2)-\dot{v}_{\mathrm{A}}{ }^{n}(j+2, k)\right\} \\
& -c_{24}\left\{\dot{w}_{\mathrm{A}}{ }^{n}(j+3, k+1)-\dot{w}_{\mathrm{A}}{ }^{n}(j+1, k+1)\right\},
\end{aligned}
$$

where $j$ and $k$ are the numbers of numerical points in the $y$ and $z$ directions, respectively. The suffixes $\mathrm{A}$ and $\mathrm{B}$ indicate the variables of circuits $\mathrm{A}$ and $\mathrm{B}$, respectively, $n$ is the time step, $\Delta t$ is the discrete time interval, and the distances of the spatial grids $\Delta y$ and $\Delta z$ satisfy $\Delta y=\Delta z=$ $\Delta d$. The quantity $c_{j k}$ is the stiffness tensor, where the suffixes $j$ and $k$ index the elements of matrix $[c]$. Note that if we use the staggered lattice for isotropic solids shown in Fig. 1(b), the third term on the right-hand side of Eq. (3) cannot be expressed.

The first equation of Newton's second law of motion for circuit $\mathrm{A}$ is discretized as

$$
\begin{aligned}
\rho \frac{2 \Delta d}{2 \Delta t} & \left\{\dot{v}_{\mathrm{A}}^{n}(j, k)-\dot{v}_{\mathrm{A}}^{n-2}(j, k)\right\} \\
= & \left\{T_{2 \mathrm{~A}}^{n-1}(j+1, k)-T_{2 \mathrm{~A}}^{n-1}(j-1, k)\right\} \\
& +\left\{T_{4 \mathrm{~A}}^{n-1}(j, k+1)-T_{4 \mathrm{~A}}^{n-1}(j, k-1)\right\},
\end{aligned}
$$

while that for circuit $\mathrm{B}$ is derived as

$$
\begin{aligned}
\rho \frac{2 \Delta d}{2 \Delta t} & \left\{\dot{v}_{\mathrm{B}}^{n}(j+1, k+1)-\dot{v}_{\mathrm{B}}^{n-2}(j+1, k+1)\right\} \\
= & \left\{T_{2 \mathrm{~B}}^{n-1}(j+2, k+1)-T_{2 \mathrm{~B}}^{n-1}(j, k+1)\right\} \\
& +\left\{T_{4 \mathrm{~B}}^{n-1}(j+1, k+2)-T_{4 \mathrm{~B}}^{n-1}(j+1, k)\right\} .
\end{aligned}
$$

The other differential equations are formulated in the same manner as Eqs. (8)-(11).

With this formulation, we alternatively calculate stresses and particle velocities with each time step $\Delta t$.

\section{MODEL PROBLEMS FOR VALIDATING METHODS USED OF IMPLEMENTING INITIAL AND BOUNDARY CONDITIONS}

In this section, we consider the implementation of initial and boundary conditions for the staggered lattice shown in Fig. 1(a).

The first model problem is that of plane wave propagation. Figure 5 shows the slowness curves on the

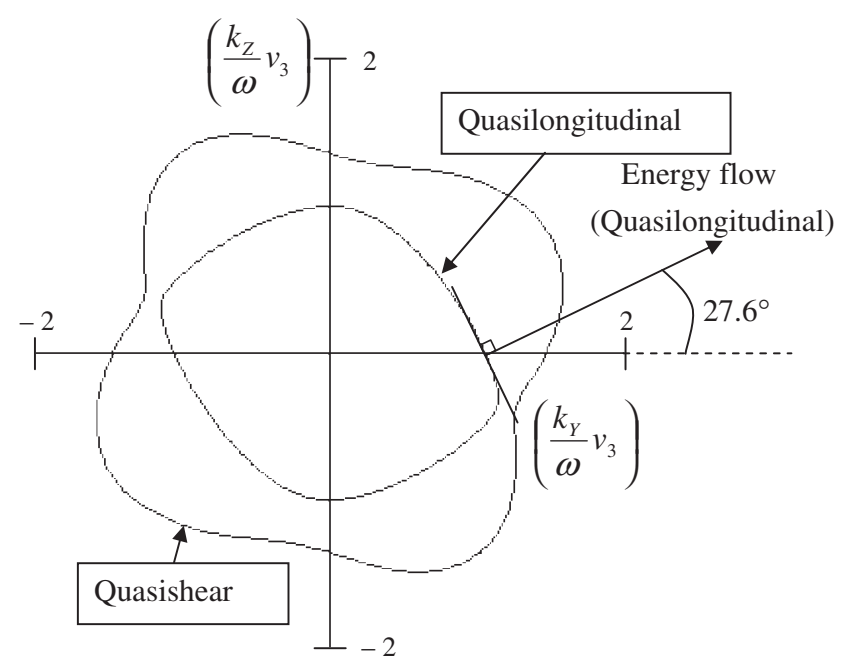

Fig. 5 Slowness curves on the $Y-Z$ plane of quartz (the piezoelectric effect is ignored).

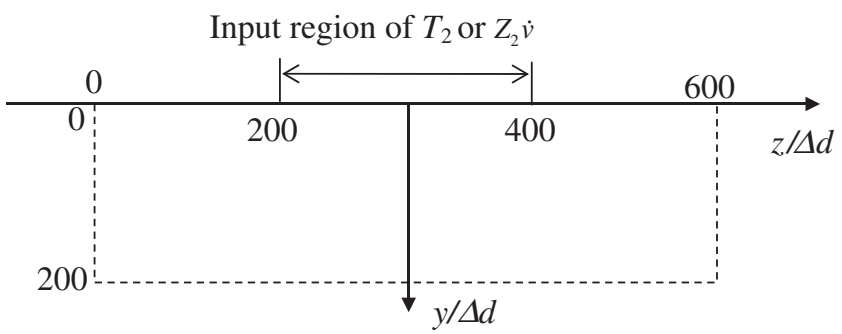

Fig. 6 Analysis model for calculating the propagation of elastic plane waves on the $Y-Z$ plane of quartz. The wave number vector of the wave is in the direction of increasing $y$.

$Y-Z$ plane of quartz [8] (the piezoelectric effect is omitted). The curves of quasi-longitudinal and quasi-shear waves are clearly noncircular; from this, we deduce that the direction of the wave number vector differs from that of the energy flow. Then we select a wave number vector with a positive $y$ component. The energy flow of the quasi-longitudinal plane wave is at angles of $\varphi=+27.6^{\circ}$ with respect to the $y$ axis, which is shown by the arrow in Fig. 5 .

Figure 6 shows the simple model problem used to test whether the FDTD formulation correctly captures the behavior of the plane waves indicated in [8]. We consider the loading of the continuous sinusoidal stress $T_{2}$ or particle velocity $Z_{2} \cdot \dot{v}$, where $Z_{2}=\sqrt{\rho c_{22}}$ is a characteristic acoustic impedance of a plane wave that has a wave number vector in the $y$ direction, on the upper surface of the analytical region between $200 \Delta d$ and $400 \Delta d$. The period of the input stress is $80 \Delta t$. Here, $\Delta t$ is a discrete time interval and assuming that $v_{33} /(\Delta d / \Delta t)=0.5$ where $v_{33}=\sqrt{c_{33} / \rho}$ is a phase velocity of the plane wave with a wave number vector in the $z$ direction and $\Delta d$ is a discrete distance.

Figure 7 indicates the distribution of the stress $T_{2}$ in 


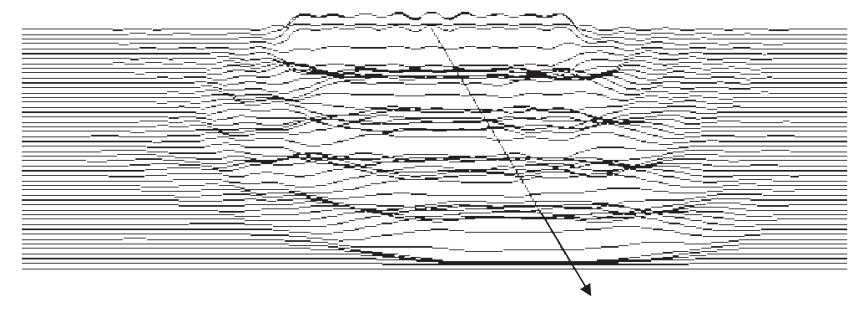

Fig. 7 Stress distributions of circuit A obtained as numerical solutions to the model problem shown in Fig. 6. The arrow in the figure indicate the direction of the energy flow deduced in Fig. 5. Input sources are set on both the $T_{2 \mathrm{~A}}$ nodes of circuit A and the $\dot{v}_{\mathrm{B}}$ nodes of circuit B.

circuit A after 400 time steps for the case when the input signals $T_{2 \mathrm{~A}}$ and $Z_{2} \cdot \dot{v}_{\mathrm{B}}$ are loaded on circuits $\mathrm{A}$ and $\mathrm{B}$, respectively. The arrow in the figure indicates the direction of the energy flow deduced from the angles shown in Fig. 5. The amplitudes of $T_{2 \mathrm{~A}}$ and $Z_{2} \cdot \dot{v}_{\mathrm{B}}$ are equal to one. The lines in Fig. 7 are drawn from $\left(0, z_{1}+c \cdot T_{2}\left(0, z_{1}\right)\right)$ to $\left(600, z_{1}+c \cdot T_{2}\left(600, z_{1}\right)\right)$, where $z_{1}$ is a discretized $z$ variable and $c$ is an arbitrary constant. The $T_{2}$ distributions on circuits $\mathrm{A}$ and $\mathrm{B}$ almost coincide with the average $T_{2}$ value on these circuits. The maximum $T_{2}$ value is close to unity.

We also investigate the $T_{2}$ distribution when the input $T_{2 \mathrm{~A}}$ signals are loaded only on circuit A. Figures 8(a) and (b) show the $T_{2}$ distributions on circuits $\mathrm{A}$ and $\mathrm{B}$, respectively. The wave shown in Fig. 8(a) seems to propagate in the direction of the wave number, and diffraction is observed at the edges of the wave. In Fig. 8(b), two waves generated on both sides of the input signal region propagate cylindrically. In Fig. 8(c), which shows the average $T_{2}$ values of circuits $A$ and $B$, the angles of wave propagations are in excellent agreement with the analytically obtained angles, but the wave amplitudes are about one-half of the analytically obtained amplitudes.

The configuration of the second model problem is shown in Fig. 9. This model is used to validate the present treatment of a free-boundary condition by FDTD analysis for an elastically anisotropic solid.

From the circuit shown in Fig. 3, it is considered important to apply the boundary condition on both circuits $\mathrm{A}$ and B. Figures 10(a) and (b) show the free-boundary conditions configured for circuits A and B, respectively. A two-dimensional $Y-Z$ plane strain condition applied to an area of $600 \Delta d \times 300 \Delta d$ is considered. The upper surface is free, while the other surfaces have no special boundary conditions because the numerical calculation is terminated before waves arrive on these surfaces.

The particle velocities $\dot{w}$ are loaded by the input signal on the free surfaces of both circuits A and B, as indicated by arrows in Fig. 10. In Fig. 9, the numbers on the arrows show the amplitudes of the input signals. The wave form of

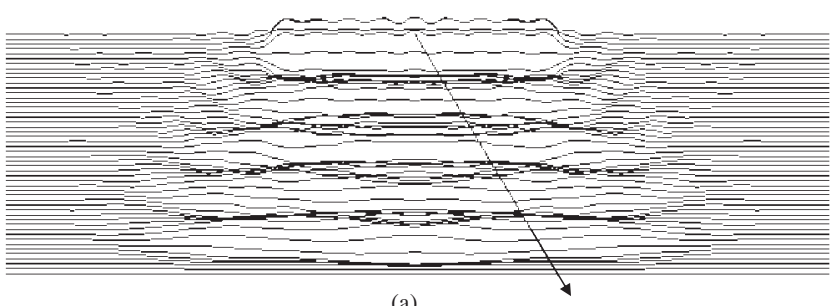

(a)
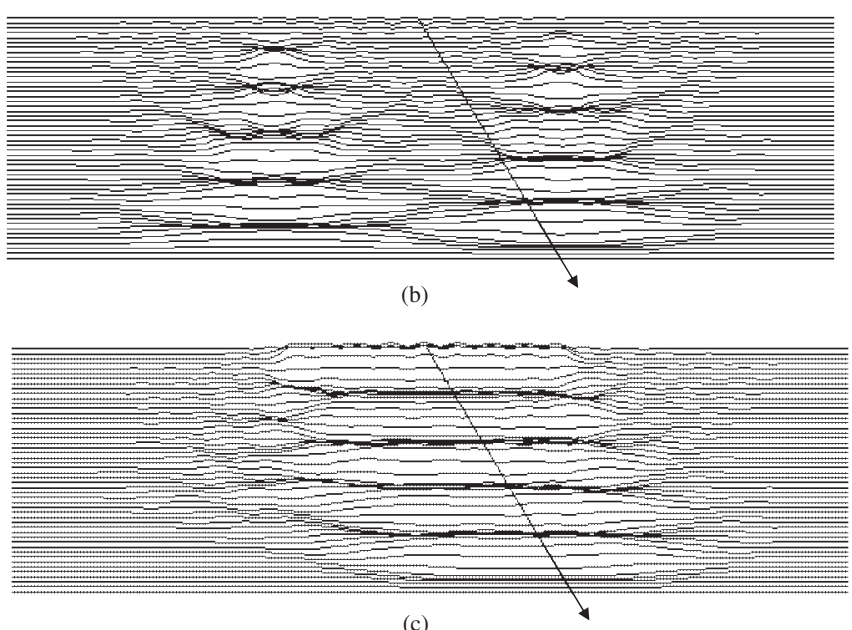

(c)

Fig. $8 T_{2}$ distribution calculated for the model problem shown in Fig. 6. Input sources are set only on the $T_{2 \mathrm{~A}}$ nodes of circuit A. (a) $T_{2}$ distribution on the nodes of circuit A. (b) $T_{2}$ distribution on the nodes of circuit B. (c) $T_{2}$ distribution given by the average $T_{2}$ values of circuits $\mathrm{A}$ and $\mathrm{B}$.

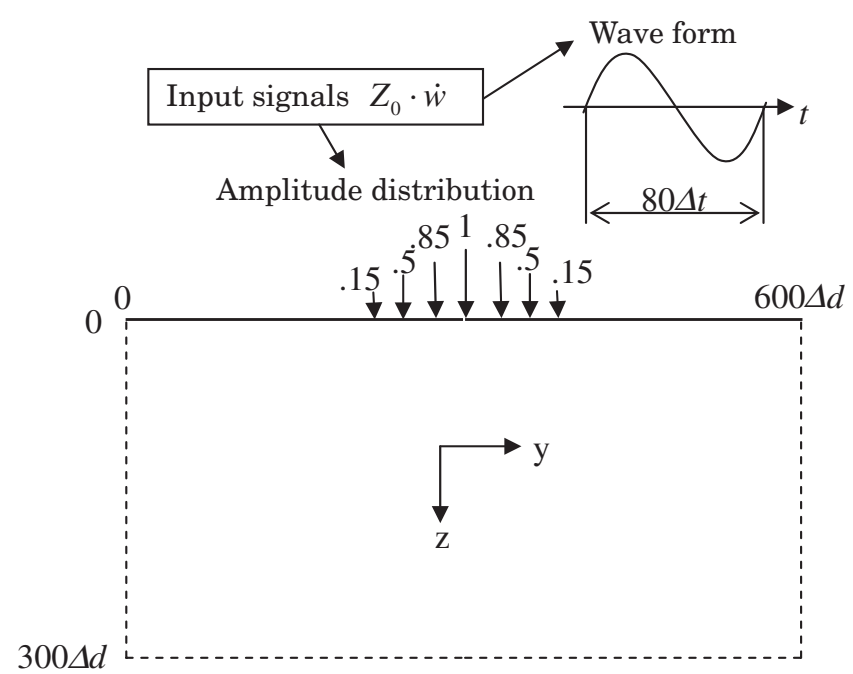

Fig. 9 Model problem used to confirm the validity of the method of implementing free-boundary conditions on an anisotropic solid under the conditions of twodimensional plane strain. The arrows on the free surface indicate the distribution of the amplitude of the input signals, and the wave form is also shown near the arrows.

the input signal is given by a single sinusoidal cycle whose time period is $80 \Delta t$.

Figure 11 shows the corresponding numerical solution 


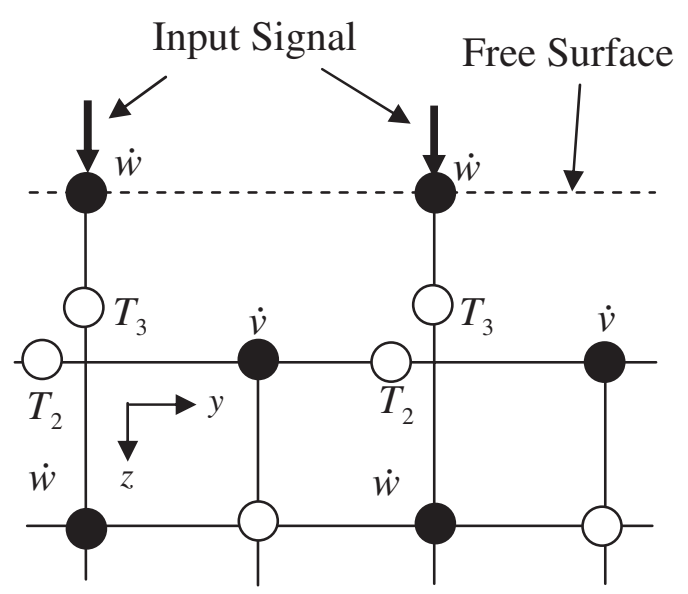

(a) Circuit A.

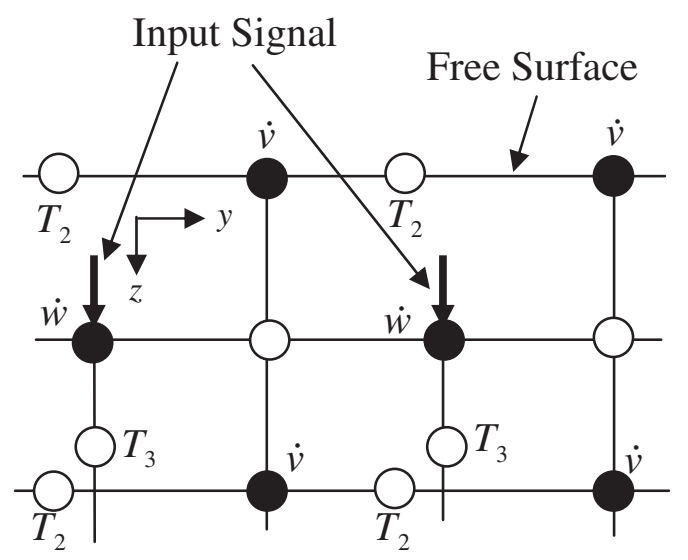

(b) Circuit B.

Fig. 10 Representation of the method of implementing free-boundary conditions.

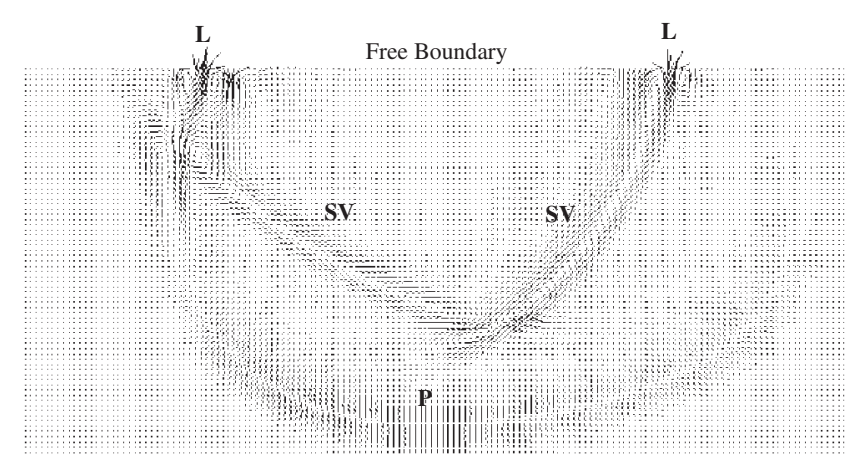

Fig. 11 Numerical solution for the particle velocity field for the model problem shown in Fig. 9. All the short lines in the figure are drawn from the discrete point $(y, z)$ to $\left(y+\left(a \cdot \dot{v}_{y}\right), z+\left(a \cdot \dot{v}_{z}\right)\right)$, where $\boldsymbol{v}=\left(\dot{v}_{y}, \dot{v}_{z}\right)$ is the particle velocity vector at the point. The constant ' $a$ ' is an arbitrary value chosen for easy understanding of the spatial pattern of the particle velocity field.

for this model problem obtained after the calculation of 600 time steps. Each line is drawn from the discrete point $(y, z)$ to $(y+(a \cdot \dot{v}), z+(a \cdot \dot{w}))$, where $\dot{\boldsymbol{u}}=(\dot{v}, \dot{w})$ is the particle velocity vector at the point. The constant ' $a$ ' is an arbitrary value chosen for easy understanding of the spatial pattern of the particle velocity field. It is clear from the results shown in Fig. 11 that the generated longitudinal waves, P, shear waves, SV, and surface acoustic waves, L, propagate through the medium. The forms of these waves on the right-hand side of the wave field differ from those on the left-hand side due to the elastic anisotropy of quartz. This result is in good agreement with the wave field calculated with the same conditions of this analysis model by the other numerical method, spatial network method [9]. We did not perform any calculations for the case when the freeboundary condition is applied to only one of the two circuits, since this situation is not physically feasible.

\section{CONCLUSION}

Methods of treating initial and boundary conditions when applying the FDTD numerical method to the analysis of elastic wave fields in anisotropic solids have been considered with reference to an electrical circuit analogy.

It was found that the staggered lattice of the FDTD analysis of anisotropic solids is expressed by the superposition of two electric circuit networks of identical configurations. One of these circuits is translated relative to the other by one grid length in both the $y$ and $z$ directions, and is then superposed onto the other circuit to yield the final overall circuit. The two circuits are connected by the mutual capacitance between the capacitances loaded at the $T_{2}$ nodes of one of the circuits and the $T_{4}$ nodes of the other circuit, which are positioned on the same stress nodes.

When the distance between the nodes of the circuit converges to zero, the average of the variables of the two circuit networks approximates the constitutive differential equations of elastic wave fields of anisotropic solids. It can be concluded that the initial and boundary conditions should be applied to both the circuit networks.

The theory was confirmed by considering two model problems: First, the method of implementing initial conditions was tested by analyzing the propagation characteristics of a plane wave that had a wave number vector lying in the positive $y$-direction. We then tested the method of implementing free-boundary conditions. In both cases, the numerical solutions obtained were in good agreement with the corresponding analytical solutions.

The author is now considering the application of the FDTD formulation to various anisotropic solids.

\section{REFERENCES}

[1] M. Sato, FDTDhou ni yo ru Danseishindou-Hadou no Kaiseki Nyumon (Morikita Syuppan, Tokyo, 2003).

[2] Y. Yamada and M. Sato, "Application of extended finitedifference time-domain method to two dimensional dynamic 
analysis of a piezoelectric vibrator," Jpn. J. Appl. Phys., 37, 255-256 (1998).

[3] H. Yanna and M. Sato, "FDTD formulation for analysis of elastic wave fields in cylindrical coordinates," Simulation, 20, 60-68 (2001).

[4] M. Sato, Y. Takahata, M. Tahara and I. Sakagami, "Expression of contour vibration modes of a square plate by scalar and vector potentials," Acoust. Sci. \& Tech., 23, 346-349 (2002).

[5] M. Sato, "Use of velocity potentials in the definition of absorbing boundaries for FDTD analysis of elastic wave fields," Acoust. Sci. \& Tech., 24, 415-418 (2003).
[6] M. Sato, "Formulation of the FDTD method for separating the particle velocity vectors of an elastic wave field into longitudinal and shear wave components," Acoust. Sci. \& Tech., 25, 382-385 (2004).

[7] M. Sato, "Numerical formulation of the FDTD method for transient analysis of elastic wave fields in the $Y-Z$ plane of quartz," Jpn. J. Appl. Phys., 44, 4490-4493 (2005).

[8] B. A. Auld, Acoustic Fields and Waves in Solids, I (John Wiley \& Sons, New York, 1973).

[9] M. Sato, "Transient analysis of anisotropic elastic media by Bergeron's method," Electron. Commun. Jpn., 72, 1-12 (1989). 